\title{
A silicon single-crystal cryogenic optical resonator
}

\author{
Eugen Wiens, Qun-Feng Chen, Ingo Ernsting, Heiko Luckmann, Ulrich Rosowski, Alexander \\ Nevsky, and Stephan Schiller* \\ Institut für Experimentalphysik, Heinrich-Heine-Universität Düsseldorf, Düsseldorf, Germany \\ *Corresponding author: Step.Schiller@uni-duesseldorf.de
}

\begin{abstract}
Compiled May 21, 2014
We report on the demonstration and characterization of a silicon optical resonator for laser frequency stabilization, operating in the deep cryogenic regime at temperatures as low as $1.5 \mathrm{~K}$. Robust operation was achieved, with absolute frequency drift less than $20 \mathrm{~Hz}$ over 1 hour. This stability allowed sensitive measurements of the resonator thermal expansion coefficient $(\alpha)$. We found $\alpha=4.6 \times 10^{-13} \mathrm{~K}^{-1}$ at $1.6 \mathrm{~K}$. At $16.8 \mathrm{~K} \alpha$ vanishes, with a derivative equal to $-6 \times 10^{-10} \mathrm{~K}^{-2}$. The temperature of the resonator was stabilized to a level below $10 \mu \mathrm{K}$ for averaging times longer than $20 \mathrm{~s}$. The sensitivity of the resonator frequency to a variation of the laser power was also studied. The corresponding sensitivities, and the expected Brownian noise indicate that this system should enable frequency stabilization of lasers at the low- $10^{-17}$ level. (C) 2014 Optical Society of America
\end{abstract}

OCIS codes: $120.3940,120.4800,140.3425,140.4780$.

Optical resonators with low sensitivity to temperature and mechanical forces are of significant importance for precision measurements in the optical and microwave frequency domain. In the optical domain, they serve to stabilize the frequencies of lasers for spectroscopic applications, notably for optical atomic clocks, and for probing fundamental physics issues such as the properties of space-time. Also, by conversion of ultrastable optical frequencies to the radio-frequency domain via an optical frequency comb, radio-frequency sources with ultralow phase noise can be realized [1], leading to e.g. radar measurements with improved sensitivity.

The conventional approach for ultra-stable optical resonators is the use of ULE (ultra-low expansion glass) material, operated at temperatures near room temperature, where the coefficient of thermal expansion (CTE) exhibits a zero crossing. While ULE resonators with optimized designs (long length, acceleration-insensitive shape) have reached impressive performance [2], their operating temperature near $300 \mathrm{~K}$ necessarily leads to a level of Brownian length fluctuations which imposes a fundamental limit to the achievable frequency stability [3], [4]. Cryogenic operation of a resonator provides one avenue towards reduction of these fluctuations. The Allan deviation of length fluctuations decreases proportional to $\sqrt{T}[3]$, if the mechanical dissipation of the resonator elements, in particular of the mirror coatings, is independent of temperature. Measurements performed thus far indicate that the dissipation of mirrors with crystalline substrates at cryogenic temperature are indeed similar to those of fused silica mirrors at room temperature [5], [6]. Nowadays, robust cryogenic solutions exist for continuous operation of even fairly large objects, such as optical resonators, at temperatures as low as $0.1 \mathrm{~K}$. This offers the possibility of reduction of resonator length fluctuations by more than one order of magnitude compared to today's lowest levels realized at room temperature, with a corresponding reduction in frequency instability of the laser stabilized to the resonator. A second outstanding feature of crystalline cryogenic optical resonators is the absence of length drift thanks to the near-perfect lattice structure.

Cryogenic optical resonators made of single-crystal sapphire have been developed early on [7] and operated at temperatures as low as $1.4 \mathrm{~K}$ [8]. Extremely small thermal expansion [7] and long-term drift were achieved [9]. Such resonators have been applied for tests of Lorentz invariance [10], local position invariance [11] and quantum space-time fluctuations [12]. Silicon, a machinable optical material available with high purity and large size, having interesting CTE properties [13], high stiffness and low mechanical dissipation [14], has first been used for an optical reference resonator by Richard and Hamilton [15]. Recently, Kessler et al. [16] developed a laser frequency stabilization system based on a vertically supported silicon resonator operated at a temperature of zero CTE, $124 \mathrm{~K}$, and achieved a high frequency stability $\left(1 \times 10^{-16}\right)$, less than $40 \mathrm{mHz}$ laser linewidth, and an extremely low long-term drift.

In this work, we present the first silicon optical resonator for absolute laser frequency stabilization operated at cryogenic temperature and discuss its thermal properties. We find that they are compatible with the goal of achieving frequency instability at the $2 \times 10^{-17}$ level.

Our silicon resonator consists of a cylindrical spacer of $250 \mathrm{~mm}$ length, $70 \mathrm{~mm}$ diameter and $15 \mathrm{~mm}$ diameter central hole. One mirror is flat, while the other has $1 \mathrm{~m}$ curvature radius. Spacer and mirror substrates were manufactured from a dislocation-free float-zone silicon crystal with [110] orientation along the cylinder axis. The substrates and spacer faces were super-polished to a residual surface roughness less than $0.1 \mathrm{~nm}$. The mirror substrates were coated with a high-reflectivity coating for $1.5 \mu \mathrm{m}$, and optically contacted to the spacer with the same orientation as in the spacer crystal. By evaluation of a ring-down of the laser power transmitted through 
the resonator at $1.5 \mathrm{~K}$, the finesse and the linewidth of the resonator were determined to be $2 \times 10^{5}$ and $3 \mathrm{kHz}$, respectively. The coupling efficiency into the resonator is $15 \%$.

A special structure for horizontal support of the resonator was implemented (see Fig. 1). It was designed for small acceleration sensitivity of the resonator's length and small stress imposed by the different CTE of resonator and supporting structure, and for blocking thermal radiation entering the cryostat through the optical window. The design is based on the concept developed in Ref. [17]. The resonator is attached to a copper frame by ten $1 \mathrm{~mm}$ diameter stainless steel wires. Their position was optimized by the finite-element method (FEM, Comsol) in order to minimize acceleration sensitivity along the three axes. The estimated sensitivities are $3 \times 10^{-10} / g$ and $3 \times 10^{-11} / g$ along the resonator optical axis and perpendicular to it, respectively. We also simulated the influence of the supporting structure on the $\mathrm{CTE}$ of the resonator. It is a function of temperature, due to the temperature dependence of the CTE of both the resonator and the frame. The results indicate negligible dependence of the CTE of the resonator $\left(\alpha_{\text {reson }}\right)$ on a change in frame temperature at temperatures below $16.8 \mathrm{~K}$. The CTE zero crossing points at $17 \mathrm{~K}$ and $124 \mathrm{~K}$ are shifted by $2 \mathrm{mK}$ and $10 \mathrm{mK}$, respectively, due to the contribution from the frame.

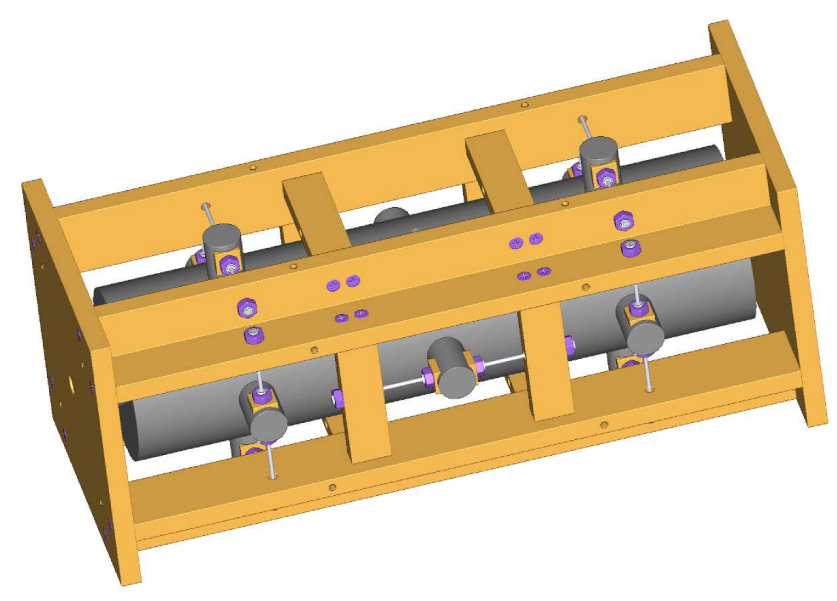

Fig. 1. Schematic of the copper support structure (yellow) with mounted silicon resonator (grey).

The frame was rigidly attached to an optical breadboard inside a cryostat. A $1.5 \mu \mathrm{m}$ fiber laser was coupled to the resonator through an optical window. The frequency was stabilized to a $\mathrm{TEM}_{00}$ mode of the resonator using the Pound-Drewer-Hall (PDH) technique [18], with a standard optical scheme and using a waveguide phase modulator. The laser power circulating inside the resonator was stabilized by detection of the light transmitted through the resonator and applying a feedback signal to an acousto-optical modulator in the optical setup.

The resonator unit was installed inside a lowvibration, two-stage pulse tube cooler cryostat with
Joule-Thomson stage. It allowed a lowest operating temperature of $1.4 \mathrm{~K}$ and a cooling power of $20 \mathrm{~mW}$. Two calibrated Cernox sensors (inaccuracy less than $10 \mathrm{mK}$ at temperatures below $30 \mathrm{~K}$ ) were available for controlling and/or monitoring the temperature of the supporting breadboard and of the resonator. One sensor was attached to the center of the breadboard, while the other was attached to the upper central part of the resonator. An AC resistance bridge was used to read out the temperature sensors. A resistance heater installed on the center of the breadboard was used for setting and maintaining a desired operating temperature. When the temperature of resonator was stabilized by controlling the resistance of the resonator sensor, the measured instability at the sensor location was $3.6 \times 10^{-5} \mathrm{~K}$ at $\tau=1 \mathrm{~s}$ averaging time, dropping as $3.6 \times 10^{-5} K /(\tau / 1 s)^{1 / 2}$ for $\tau$ up to $10^{4} \mathrm{~s}$. We estimate that the temperature instability of the average temperature of the resonator is within a moderate factor of the above number, since the very high thermal diffusivity of silicon at cryogenic temperature allows for a rapid thermal equilibration within the resonator. The AC bridge electronics contributes a specified systematic error of $1.7 \times 10^{-5} \mathrm{~K}$ per degree variation of the ambient temperature. Thus, in a laboratory stabilized to $0.5 \mathrm{~K}$, the resonator temperature systematic shift is less than $1 \times 10^{-5} \mathrm{~K}$.

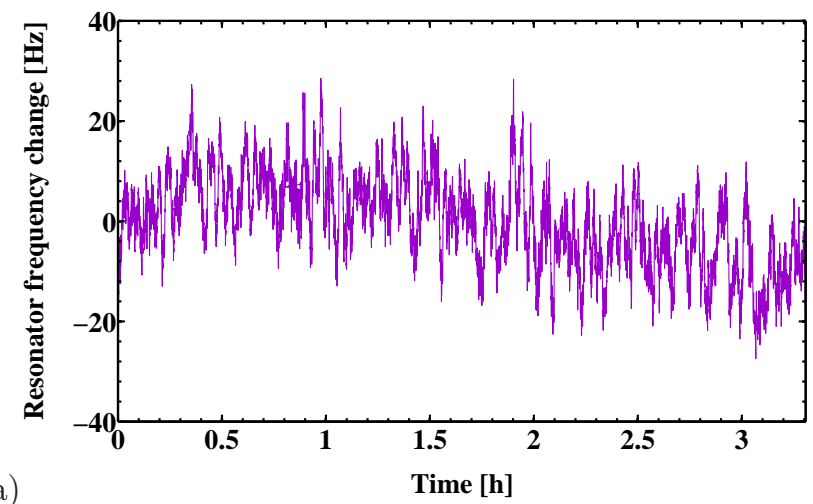

a)

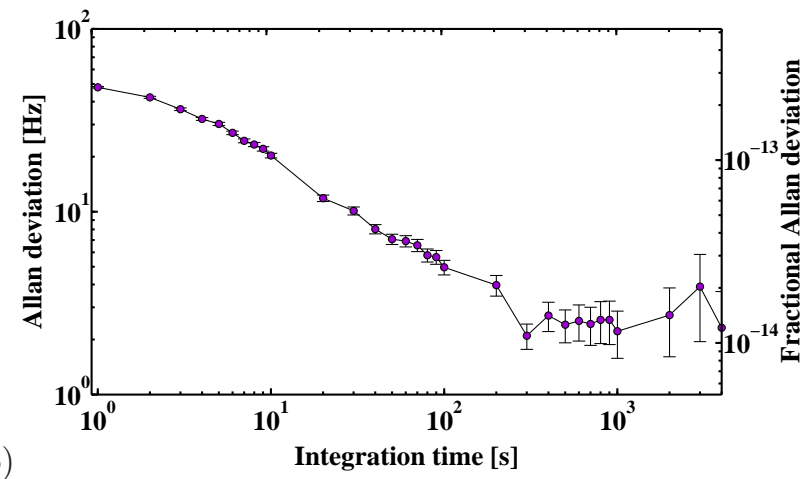

Fig. 2. a) Time trace averaged over $60 \mathrm{~s}$ of a typical resonator frequency measurement relative to a hydrogen maser, b) corresponding Allan deviation.

Measurements of the absolute optical resonator frequency were done by measuring the frequency of the laser 
stabilized to it with respect to a hydrogen maser, using a commercial erbium-doped fiber laser frequency comb. A comb mode near $1064 \mathrm{~nm}$ was phase-locked to a $1064 \mathrm{~nm}$ optical reference having $2 \times 10^{-15}$ short-term frequency instability. This reduced significantly the linewidth of comb modes in the $1.5 \mu \mathrm{m}$ spectral range and permitted achieving a beat note between the Si-resonator-stabilized laser and the comb with a linewidth as low as $1.7 \mathrm{kHz}$. The laser light was sent from the laser to the comb via a $150 \mathrm{~m}$ long fiber. A typical resonator frequency time trace and the corresponding instability, with all parameters including resonator temperature held constant, is depicted in Fig. 2. For integration times longer than $1000 \mathrm{~s}$ the frequency instability was approximately $3 \mathrm{~Hz}$ $\left(2 \times 10^{-14}\right)$. The contributions to this instability will be subject of future investigations.

Given this level of absolute frequency instability and the good resolution of the temperature sensors, the resonator's thermal expansion coefficient could be accurately measured. The measurements were done either by heating the resonator or by letting it cool down to $1.5 \mathrm{~K}$ after heating it up to a desired temperature. The rate of temperature change was approximately $1.5 \mathrm{~K} / \mathrm{h}$ at temperatures above $1.8 \mathrm{~K}$ and much lower for temperatures below $1.8 \mathrm{~K}$, where it was limited by the cooling capacity of the cryostat. No significant discrepancies were found between the two measurement procedures.

The temperature dependence of the resonator frequency is presented in Fig. 3. The total change in frequency from $1.5 \mathrm{~K}$ to $23.8 \mathrm{~K}$ is $6 \mathrm{MHz}$, with most of the shift occurring in the region above $20 \mathrm{~K}$. A minimum of the frequency occurs at $T_{\alpha=0}=16.81 \mathrm{~K}$ with negative frequency derivative $d f / d T$ (length expansion) below this temperature and positive (length contraction) above it [19]. Between $1.6 \mathrm{~K}$ and $2 \mathrm{~K}$ the shift in frequency is only $100 \mathrm{~Hz}\left(5 \times 10^{-13}\right)$.

Whereas the CTE of an insulating crystal at low temperatures is expected to be positive and proportional to $T^{3}$ [13], in the case of silicon its thermal expansion behaviour (expansion turns into contraction between $16.8 \mathrm{~K}$ and $124 \mathrm{~K}$ ) necessarily implies that such a dependence can accurately hold only for $T \rightarrow 0$. The value predicted from compressibility and heat capacity measurements is $\alpha_{S i}=4.8 \times 10^{-13} \mathrm{~K}^{-1}(T / \mathrm{K})^{3}$ [13]. As displayed in Fig. 3, a pure $T^{3}$ dependence does not fit the data well. Therefore, the data were fitted with a function containing additional terms. At the lowest temperatures, we find $\alpha_{\text {reson }}(T \rightarrow 0)=\left(1.1 T^{3}-7.8 T^{4}+8.4 T^{5}\right) \times 10^{-13} \mathrm{~K}^{-1}$, where $T$ is the temperature in Kelvin. Our measured $\alpha_{\text {reson }}$ is thus lower then the predicted value for bulk silicon at temperatures below $3 \mathrm{~K}$. At $1.6 \mathrm{~K}$, the value is $\alpha_{\text {reson }}=4.6 \times 10^{-13} / \mathrm{K}$.

The frequency data around the frequency minimum at $T_{\alpha=0}=16.8 \mathrm{~K}$ were accurately fitted by a fourth-order polynomial from which we obtain the resonator expansion coefficient $\alpha_{\text {reson }}\left(T \simeq T_{\alpha=0}\right)=\sum_{n=0}^{2} B_{n}(T / \mathrm{K})^{n}$ with $B_{n}=\left(37 \times 10^{-9},-4.94 \times 10^{-9}, 1.64 \times 10^{-10}\right) \mathrm{K}^{-1}$. The complete dataset from $1.6 \mathrm{~K}$ to $23.8 \mathrm{~K}$ was fit-

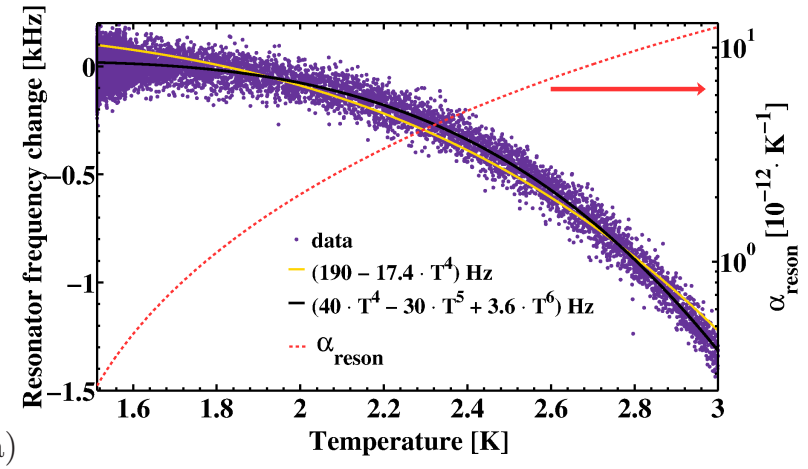

a)

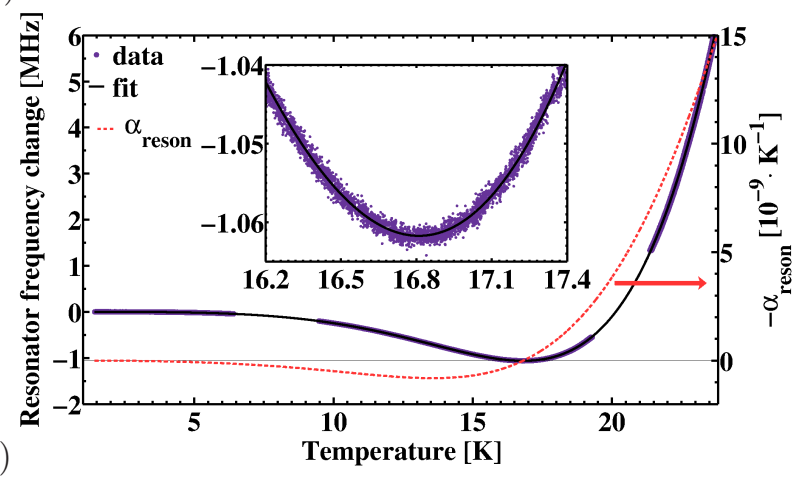

Fig. 3. Temperature dependence of the silicon resonator frequency in two temperature intervals. a) Interval $1.5 \mathrm{~K}$ to $3 \mathrm{~K}$, with fit results in the legend and corresponding expansion coefficient $\alpha_{\text {reson }}$ calculated from the second fit in the legend. b) Interval from $1.5 \mathrm{~K}$ to $23.8 \mathrm{~K}$ with a polynomial fit and calculated expansion coefficient $\alpha_{\text {reson }}$. The inset in b) shows a zoom of the interval where the CTE crosses zero.

ted with a ninth-order polynomial (Fig. 3b), from which we obtain the resonator thermal expansion coefficient $\alpha_{\text {reson }}(T)$ shown in Fig. 3 b). It is given by $\alpha_{\text {reson }}(T)=\sum_{n=0}^{8} A_{n}(T / K)^{n}$, with $A_{n}=(-9 \times$ $10^{-22}, 4 \times 10^{-20},-7 \times 10^{-19}, 2 \times 10^{-17},-3 \times 10^{-16}, 2 \times$ $\left.10^{-15},-1 \times 10^{-14}, 3 \times 10^{-14},-2 \times 10^{-14}\right) \mathrm{K}^{-1}$.

To our knowledge, only two measurements of thermal expansion of silicon at cryogenic temperature, down to $12 \mathrm{~K}$ and $6 \mathrm{~K}$ [13] were performed previously. However, due to the difficulty of measuring the corresponding small CTEs, accurate CTE values were only given above $13 \mathrm{~K}$ [13], where they are larger than $1 \times 10^{-9} / \mathrm{K}$. Our value of $T_{\alpha_{\text {reson }}=0}=16.8 \mathrm{~K}$ is similar, but not identical to that of Ref. [13], where $17.8 \mathrm{~K}$ was measured. Our expansivity temperature derivative at $16.8 \mathrm{~K}$ is $d \alpha_{\text {reson }}\left(T_{\alpha_{\text {reson }}=0}\right) / d T=-6.0 \times 10^{-10} / \mathrm{K}^{2}$, which is comparable to $-8.9 \times 10^{-10} / \mathrm{K}^{2}$ at $17.8 \mathrm{~K}$ in Ref. [13]. This expansivity derivative at $16.8 \mathrm{~K}$ is nearly a factor 20 smaller than the one at the second zero-CTE temperature $124.2 \mathrm{~K}, 1.7 \times 10^{-8} / \mathrm{K}^{2}$ [16]. This means that $16.8 \mathrm{~K}$ could also be a candidate operating temperature, with the advantage of less temperature sensitivity, possibly smaller thermal noise, as compared to $124 \mathrm{~K}$, and less stringent requirements on cryostat construction. As 
mentioned above, the mirror coating dissipation at this temperature is similar to the room-temperature values for conventional mirror substrates.

With an estimated upper limit of $1 \times 10^{-4} \mathrm{~K}$ resonator temperature instability at $1 \mathrm{~s}$ (discussed above) and the measured thermal expansion $\alpha_{\text {reson }}(T=1.6 \mathrm{~K})=$ $4.6 \times 10^{-13} \mathrm{~K}^{-1}$, the corresponding fractional frequency instability is about $5 \times 10^{-17}$ at $1 \mathrm{~s}$ and drops to $1 \times 10^{-17}$ at $\tau=30 \mathrm{~s}$. The fractional frequency shift error due to $\mathrm{AC}$ bridge systematics is expected to be less than $1 \times 10^{-17}$.

Fluctuations of the laser power incident on the resonator introduce changes in the power dissipated on the resonator mirrors and result in unwanted fluctuations in resonator length due to the thermal expansion of the mirrors and of the spacer. We measured the resonator frequency change caused by a varying level of intraresonator power at two different operating temperatures. We could not observe any effect, setting an upper limit of $20 \mathrm{~Hz}$ change for a $30 \%$ power change, both at $1.6 \mathrm{~K}$ and at $16.8 \mathrm{~K}$. This upper limit corresponds to approximately $3 \times 10^{-14} / \mu \mathrm{W}$.

Because of the absence of measurable effect, we also simulated the effect of laser heating using FEM and found a $1.4 \mathrm{fm}$ mirror distance change for $10 \mu \mathrm{W}$ power dissipated on each mirror at $1.5 \mathrm{~K}$, i.e. a fractional resonator frequency change of $3 \times 10^{-16} / \mu \mathrm{W}$ total dissipated power. The simulation also shows that thermal equilibrium of the heated mirrors and the spacer is reached with a time constant of approximately $1 \mathrm{~s}$ in case of good thermal contact with the breadboard. For our experiment, the power level dissipated in the resonator is estimated as $1.5 \mu \mathrm{W}$. If the transmitted laser power is actively stabilized to $1 \%$ or better, a level that is expected to be well feasible using an advanced photodetector, the corresponding fractional frequency fluctuations would be $5 \times 10^{-18}$ or less.

In summary, we demonstrated a silicon optical resonator for laser frequency stabilization that can be operated at temperatures between $1.5 \mathrm{~K}$ and $24 \mathrm{~K}$ and investigated its thermal properties. Stable, long-term operation was achieved at temperature as low as $1.4 \mathrm{~K}$. The support structure for the resonator allows to take advantage of the low CTE of bulk silicon. The thermal expansion measurement was performed both at the lowest absolute temperature and with the highest sensitivity for a silicon object so far. The influence of finite thermal expansion coefficient and residual temperature instability on the cavity frequency was determined to be at the $5 \times 10^{-17}$ fractional level and below, depending on averaging time. The sensitivity to changes in circulating laser power is expected to be controllable to the level better than $1 \times 10^{-17}$ using an appropriate power stabilization unit. For this resonator, at the temperature $1.6 \mathrm{~K}$, the expected total Brownian noise-induced frequency instability is calculated to be $6 \times 10^{-18}$, assuming a coating loss angle $\varphi=1 \mathrm{mrad}$, as determined for silicon mirrors at $20 \mathrm{~K}$ in Ref. [6].
We conclude that the thermal properties of the described system should allow to stabilize the frequency of a laser to an instability of less than $2 \times 10^{-17}$ for integration times larger than $30 \mathrm{~s}$, taking into account that the instability arising from the laser frequency locking system itself can be reduced to the level of $1 \times 10^{-17}$ [20].

We are very grateful to Timo Müller (Wacker Chemitronic) for providing the crystal and to T. Legero and U. Sterr (PTB) for important help on the resonator. This work has been funded by ESA project no. 4000103508/11/D/JR. We thank I. Zayer and J. de Vicente for support.

\section{References}

1. Y. Y. Jiang, A. D. Ludlow, N. D. Lemke, R. W. Fox, J. A. Sherman, L.-S. Ma, and C. W. Oates, Nat. Phot. 5, 158-161 (2011).

2. T. L. Nicholson, M. J. Martin, J. R. Williams, B. J. Bloom, M. Bishof, M. D. Swallows, S. L. Campbell, and J. Ye, Phys. Rev. Lett. 109, 230801 (2012).

3. K. Numata, A. Kemery, und J. Camp, Phys. Rev. Lett. 93, 250602 (2004).

4. G. D. Cole, W. Zhang, M. J. Martin, J. Ye, M. Aspelmeyer, Nature Photonics 7, 644-650 (2013).

5. K. Yamamoto, S. Miyoki, T. Uchiyama, H. Ishitsuka, M. Ohashi, K. Kuroda, T. Tomaru, N. Sato, T. Suzuki, T. Haruyama, A. Yamamoto, T. Shintomi, K. Numata, K. Waseda, K. Ito, und K. Watanabe, Phys. Rev. D 74, 022002 (2006).

6. M. Granata, K. Craig, G. Cagnoli, C. Carcy, W. Cunningham, J. Degallaix, R. Flaminio, D. Forest, M. Hart, J.-S. Hennig, J. Hough, I. MacLaren, I. W. Martin, C. Michel, N. Morgado, S. Otmani, L. Pinard, und S. Rowan, Opt. Lett. 38, 5268-5271 (2013).

7. S. Seel, R. Storz, G. Ruoso, J. Mlynek, und S. Schiller, Physical Review Letters 78, 4741-4744 (1997).

8. R. Storz, Dissertation, Univ. Konstanz (1998).

9. R. Storz, C. Braxmaier, K. Jack, O. Pradl, und S. Schiller, Optics Letters 23, 1031-1033 (1998).

10. P. Antonini, M. Okhapkin, E. Goklü, S. Schiller, Physical Review A 71, 050101, (2005).

11. C. Braxmaier, H. Müller, O. Pradl, J. Mlynek, A. Peters, S. Schiller, Physical Review Letters 88, 010401 (2001).

12. S. Schiller, C. Lammerzahl, H. Müller, C. Braxmaier, S. Herrmann, A. Peters, Physical Review D 69, 027504 (2004).

13. K. G. Lyon, G. L. Salinger, C. A. Swenson, and G. K. White, J. Appl. Phys. 48, 865 (1977).

14. D. F. McGuigan, C. C. Lam, R. Q. Gram, A. W. Hoffman, D. H. Douglass, H. W. Gutche, J. Low Temp. Phys. 30, 621-629 (1978).

15. J.-P. Richard, J. J. Hamilton, Rev. Sci. Instr. 62, 23752378 (1991).

16. T. Kessler, C. Hagemann, C. Grebing, T. Legero, U. Sterr, and F. Riehle, Nature Photonics 6, 687, (2012).

17. U. Sterr, German patent DE 102011015 489.2, (2011).

18. R. W. P. Drever, J. L. Hall, F. V. Kowalski, J. Hough, G. M. Ford, A. J. Munley, and H. Ward, Appl. Phys. B 31, 97 (1983). 
19. T.F. Smith, G.K. White, J. Phys. C 8, 2031-2042 (1975).

20. Q.-F. Chen, A.Yu. Nevsky, and S. Schiller, Appl. Phys. B 107, 679-683 (2012). 